\title{
Cholinergic and adrenergic innervation of the pancreas in chinchilla (Chinchilla Laniger Molina)
}

\author{
Malgorzata Radzimirska, Jacek Kuchinka, \\ Elzbieta Nowak, Wojciech Trybus, Aleksander Szczurkowski
}

Department of Medical Biology, Institute of Biology, Jan Kochanowski University in Kielce, Poland

\begin{abstract}
Introduction. Cholinergic and adrenergic innervation of the pancreas in chinchilla (Chinchilla Laniger Molina) was examined in this study. The pancreas is both an exocrine and endocrine gland with autonomic and sensory innervation presented by the numerous nerve fibers and small agglomerations of nerve cells.

Material and methods. Investigations were performed on 16 adult chinchillas of both sexes. The material was collected immediately after death of the animals. Histochemical methods: AChE and SPG were used, in addition to routine technique of single and double immunohistochemical (IHC) staining using whole mount specimens and freezing sections with a thickness of 8 to $12 \mu \mathrm{m}$. In the immunofluorescence staining, primary antibodies directed against markers used to identify cholinergic - ChAT and VAChT, and adrenergic - D $\beta \mathrm{H}$ and TH neurons. Secondary antibodies were coupled to Alexa Fluor 488 and Alexa Fluor 555 fluorophores.

Results. Histochemical studies (AChE) revealed that chinchilla pancreatic cholinergic innervation consisted of ganglionic neurocytes and numerous nerve fibers. These structures are located in the parenchyma of the exocrine part of the organ in close proximity to blood vessels and are present within the walls of the pancreatic ducts and interstitial connective tissue. A delicate fiber network around the Langerhans islets was also observed. The most numerous cholinergic structures were found in the head and tail, and the least numbers were found in the body of the pancreas. The SPG method revealed that adrenergic fibers form a network in the adventitia of blood vessels, and individual fibers run throughout the pancreatic parenchyma. Moreover, adrenergic nerve fibers were observed around the ganglionic neurocytes. This innervation was similar in all parts of the investigated organ. IHC investigations allowed observations of both the cholinergic and adrenergic activities of autonomic nerve structures. Additionally, using ChAT/D $\beta \mathrm{H}$ double staining, colocalization of these substances was observed in the fibers of the pancreatic parenchyma that passed through the cholinergic ganglia. Colocalization of VAChT and $\mathrm{TH}$ was found in nerve fibers of the exocrine part, in the walls of blood vessels, and in individual nerve cells. Colocalization of ChAT/D $\beta \mathrm{H}$ and VAChT/TH was observed in the single nerve cells and in the small (2-3 cell) ganglia. ChAT- and $\mathrm{D} \beta \mathrm{H}$-immunopositive nerve fibers were found in the area of the islets of Langerhans. Conclusions. The results indicate a more intense cholinergic innervation of the chinchilla's pancreas, which is represented by both ganglia and nerve fibers, while adrenergic structures are mainly represented by fibers and only single neurocytes. This arrangement of the investigated structures in this species may imply a major role for hormonal control of exocrine secretion in rodents. (Folia Histochemica et Cytobiologica 2020, Vol. 58, No. 1, $54-60)$
\end{abstract}

Key words: chinchilla laniger; pancreas; cholinergic innervation; adrenergic innervation; histochemistry; IHC

Correspondence address: Malgorzata Radzimirska, $\mathrm{PhD}$

Department of Medical Biology, Institute of Biology,

Jan Kochanowski University in Kielce,

Uniwersytecka 7, 25-406 Kielce, Poland

e-mail: malgorzata.radzimirska@ujk.edu.pl 


\section{Introduction}

Corresponding to its important functions, the pancreas is extensively innervated by autonomic and afferent nerve fibers [1-5]. Nerve fibers reach the pancreas in the form of neurovascular stalks that follow the blood vessels also within the pancreatic tissue and end or begin close to capillary walls and endocrine cells [6].

Parasympathetic preganglionic nerve fibers running as a component of the vagal nerve reach the pancreatic ganglia, and then, as postganglionic fibers, these parasympathetic fibers innervate the pancreatic acini. The myelinated axons of the preganglionic sympathetic neurons, located in the lateral horn of the thoracic and the upper lumbar segments of the spinal cord, project to ganglionic cells in the paravertebral sympathetic ganglia or to the celiac and mesenteric ganglia via the splanchnic nerves and then supply the pancreas as postganglionic fibers $[7,8]$.

Autonomic innervation of the pancreas has been investigated in numerous vertebrate species, both in domestic animals, such as pigeon $[9,10]$, hen [11], cat [12], rabbit [12, 13], sheep [14, 15], pig [16], and the representatives of other species, such as the African silverbill $[9,10]$, and mainly rodents, such as rat $[12$, 17], mouse [5, 6, 18], or Egyptian spiny mouse [19].

The pancreatic autonomic structures in the investigated species consist of numerous nerve fibers and ganglia, located mainly in the exocrine part of the pancreas. Thin bundles of fibers around the islets of Langerhans and a few individual fibers between endocrine cells have been observed. In many animal species including humans [20], cholinergic innervation structures seem to be more intensively developed than the adrenergic structures and consist of both nerve fibers and an agglomeration of ganglionic cells, while the adrenergic fibers mainly form the delicate networks [11, 13, 15, 19].

Chinchillas were previously reared for their valuable fur, but in recent years this animal has more frequently served as a model species for research into human diseases, especially hearing dysfunction in addition to research related to digestive system and pneumonia [21]. They are also used as laboratory animals for studies of cerebral vascularization [22], innervation of the gastrointestinal system [23-25], and previously unreported morphology of the extrahepatic biliary tract [26].

The aim of this study was to describe cholinergic and adrenergic innervation in the chinchilla pancreas (Chinchilla Laniger Molina). Histochemical methods have often been used in studies of autonomic pancreatic innervation. We consider it justified to use immunohistochemistry for the study of innervation in this rodent, which will undoubtedly allow a more detailed analysis of the delicate nerve structures supplying the pancreas. Our results will provide valuable macromorphological comparative data for investigation of pancreatic innervation in different species of small mammals.

\section{Material and methods}

Investigations were carried out on sixteen adult (10 months) individuals of chinchilla (Chinchilla Laniger Molina) both sexes. Material was collected immediately after industrial slaughter in Chinchillas Fur Farm. These studies were approved in accordance with the law of Act of 15 January 2015: ,The protection of animals used for scientific or educational purpose" (studies on tissues obtained post-mortem do not require an approval of the Ethics Committee). Tissue were collected from the head, body and tail of pancreas and stained by the histochemical and immunohistochemical (IHC) methods. Histochemical investigations were performed on pancreases of eight individuals according to acetylcholinesterase (AChE) method [19, 27, 28] and SPG method [29], and pancreases of eight animals were used for routine immunofluorescence staining.

\section{Histochemical methods}

Two types of specimens were prepared: macromorphological specimens (whole mount), and frozen specimens (Cryomatrix, Thermo Shandon, Waltham, MA, USA) and cut for $12 \mu \mathrm{m}$ sections. Next specimens were stained for cholinergic structures using the AChE method. After incubation in the staining solution (acetyl thiocholine iodide, acetate buffer, sodium citrate, copper sulfate, distilled water, potassium ferricyanide) at $37^{\circ} \mathrm{C}$, and $\mathrm{pH} 6.4-6.8$, slides were rinsed in distilled water, dehydrated and mounted in DPX. Activity of non-specific cholinesterase was blocked with iso-OMPA $\left(\mathrm{C}_{12} \mathrm{H}_{32} \mathrm{~N}_{4} \mathrm{O}_{3} \mathrm{P}_{2}\right.$ tetraisopropylpyrophosphoramide). For the detection of adrenergic nerve fibers the sucrose-phosphate-glyoxylic acid method was used (SPG solution: sucrose, potassium phosphate monobasic and glyoxylic acid monohydrate). The unfixed whole mount specimens and frozen section were dipped immediately in SPG solution for $5 \mathrm{sec}$, and dried under a strong stream of air for $10 \mathrm{~min}$. Subsequently, sections were covered with a drop of light mineral oil and heated to $95^{\circ} \mathrm{C}$ for 3 min and cover-slipped. The histochemically stained specimens were observed using fluorescence microscope Nikon Eclipse 90i (Nikon, Tokyo, Japan) and digital pictures were taken with Nikon Digital Sight SD-L1 system, and Nis -Elements 3.22 software and 60 sections were used for the measurement. 
Table 1. Primary antisera used in the research

\begin{tabular}{|l|c|c|c|c|c|}
\hline Antigen & Host & Type & Dilution & Catalog No. & Supplier \\
\hline ChAT & Goat & Polyclonal & $1: 100$ & NBP1-30052 & Novus Biologicals \\
\hline VAChT & Rabbit & Polyclonal & $1: 1000$ & EUD261 & Acris Antibodies \\
\hline D $\beta \mathrm{H}$ & Rabbit & Polyclonal & $1: 500$ & NBP1-78349 & Novus Biologicals \\
\hline TH & Mouse & Monoclonal & $1: 500$ & MAB318 & Millipore \\
\hline
\end{tabular}

Table 2. Secondary antisera used in the research

\begin{tabular}{|l|c|c|c|c|}
\hline Host & Fluorophore & Dilution & Catalog No. & Supplier \\
\hline Donkey anti-rabbit & Alexa Fluor 488 & $1: 500$ & A21206 & Invitrogen \\
\hline Donkey anti-goat & Alexa Fluor 555 & $1: 500$ & A21432 & Invitrogen \\
\hline Donkey anti-mouse & Alexa Fluor 555 & $1: 500$ & A31570 & Invitrogen \\
\hline
\end{tabular}

\section{Immunohistochemistry}

Animals were transcardially perfused with 0.41 of $4 \%$ ice-cold buffered paraformaldehyde ( $\mathrm{pH} 7.4)$ and pancreas was collected. The tissues were postfixed by immersion in the same fixative for $15 \mathrm{~min}$, rinsed with phosphate buffer ( $\mathrm{pH} 7.4 ; 0.1 \mathrm{M})$, transferred to and stored in $30 \%$ buffered sucrose solution ( $\mathrm{pH}$ 7.4) until further processing. The pancreas was cut into $8 \mu \mathrm{m}$ thick cryostat sections (Shandon Cryotome E; Thermo Scientific). Next, slides and whole mount specimens were stained with single and double immunohistochemical method. Tissues were incubated for $16-20 \mathrm{~h}$ at room temperature (RT) with primary antibodies against choline acetyltransferase (ChAT), vesicular acetylcholine transporter (VAChT), dopamine $\beta$-hydroxylase $(\mathrm{D} \beta \mathrm{H})$, and tyrosine hydroxylase (TH) (Table 1). Thereafter, slides were incubated with secondary fluorophore (fluorochrome)-conjugated antibodies Alexa Fluor 555 and Alexa Fluor 488 for one hour (Table 2). Finally, specimens were coversliped with buffered glycerol and analyzed under a Nikon A1R confocal microscope based on a Nikon Eclipse Ti inverted microscope (Nikon Instruments Inc., New York, NY, USA).

Control of specificity of staining was performed by preabsorption of a diluted antiserum with $20 \mu \mathrm{g} / \mathrm{ml}$ of an appropriate antigen, which abolished the specific immunoreactivity completely. In addition, experiments were carried out in which the primary antiserum was replaced by non-immune serum, or by PBS, in order to verify the specificity of particular immunoreactions.

\section{Results}

Histochemical investigation using the AChE method revealed that cholinergic innervation of the pancreas in the chinchilla consisted of numerous ganglia and nerve fibers (Fig. 1A-B). These ganglia were different in sizes and had oval or elongated shapes. Their dimensions ranged from 20.40 to 85.47 (49.77 \pm 17.98 $\mu \mathrm{m}$, mean $\pm \mathrm{SD})$ and 14.22 to $40.87(28.06 \pm 10.59$ $\mu \mathrm{m})$ micrometers in length and width, respectively. A few clearly larger ganglia with about 15 cells in the cross-section were seen to have dimensions of up to 60.07 and $113.64 \mu \mathrm{m}$ for width and length, respectively. These ganglia were located in the interstitial connective tissue, in the parenchyma of the exocrine part, and in the immediate vicinity of blood vessels and intralobular and interlobular ducts. Additionally, individual nerve cells were observed in the parenchyma of the organ. Both individual fibers and fiber bundles of different thickness were found in the parenchyma, along the pancreatic ducts, and in the wall of pancreatic ducts and along of the blood vessels. The bundles of nerve fibers varied from 39.03 to $83.84 \mu \mathrm{m}$ in thickness. Moreover, a delicate network of nerve fibers was visible around the islets of Langerhans and between endocrine cells. Most of the cholinergic structures were present in the head and tail of the pancreas with the least numerous in its body.

Using the SPG method, it was found that adrenergic fibers form a network in the adventitia wall of blood vessels (Fig. 1C-D). Additionally, this type of nerve fiber with its characteristic varicosities was visible around the ganglionic neurocytes and was observed as individual fibers in the parenchyma (Fig. 1D). The distribution of adrenergic fibers was similar in all parts of the pancreas.

Immunohistochemical investigations confirmed the results obtained with AChE and SPG methods. ChAT (Fig. 1E-G) and VAChT (Fig. 1H) were found as cholinergic markers in the nerve fibers, as well as in 

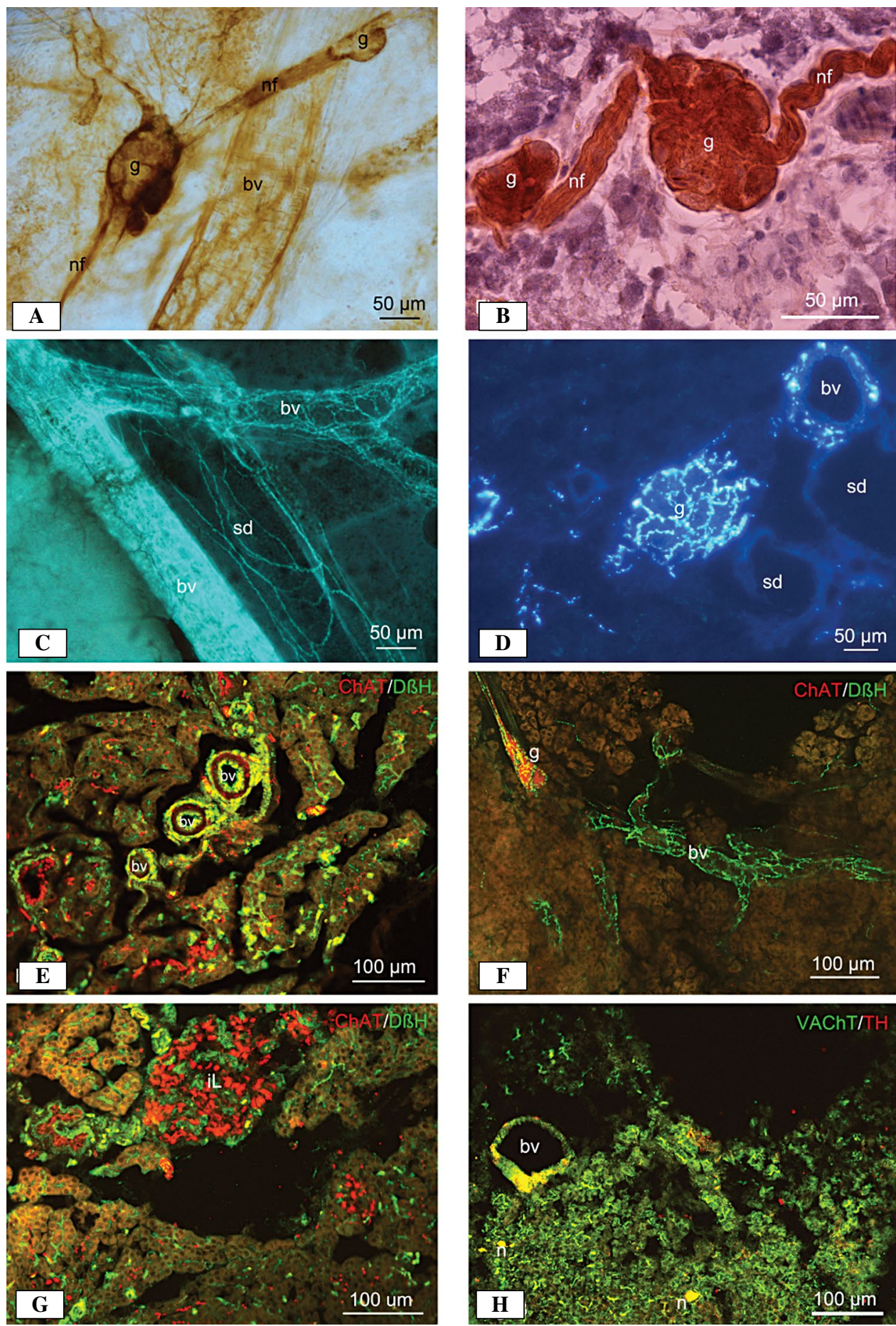

Figure 1. A. The cholinergic ganglia and nerve fibers in the parenchyma of the head of chinchilla's pancreas. The histochemical staining demonstrating AChE activity was performed as described in methods. Whole mount specimens; B. The cholinergic ganglia and nerve fibers in the parenchyma of the tail of pancreas. AChE, frozen section; $\mathbf{C}$. The network of the adrenergic fibers in the blood vessels in the parenchyma of the head of pancreas (SPG, whole mount specimens); D. The adrenergic fibers in the blood vessels, in the parenchyma, and between cells in the ganglion of the corpus of pancreas (SPG, frozen section); E. The cholinergic and adrenergic nerve fibers in parenchyma, in the wall of blood vessels of the head of pancreas (ChAT - red, D $\beta \mathrm{H}$ - green, colocalization - gold/yellow, frozen section); $\mathbf{F}$. The cholinergic ganglion in parenchyma and network of the adrenergic fibers in the wall of blood vessel of the head pancreas (ChAT - red, D $\beta \mathrm{H}-$ green, colocalization — gold/yellow, whole mount specimens); G. The cholinergic and adrenergic nerve fibers on the islet of Langerhans of the corpus of pancreas (ChAT — red, D $\beta \mathrm{H}$ - green, colocalization — gold/yellow, frozen section); H. The cholinergic and adrenergic nerve fibers in parenchyma, and in the wall of blood vessel of the tail in pancreas (VAChT - green, TH red, colocalization — gold/yellow, whole mount specimens). Abbreviations: bv — blood vessel; g — ganglion; iL — islet of Langerhans; $\mathrm{n}$ - neurocytes; $\mathrm{nf}$ - nerve fibers; sd — secretory duct 
the ganglionic neurocytes. They were located in the parenchyma of the exocrine part, around and in the wall of blood vessels, in the interlobular connective tissue, and in close vicinity to the pancreatic ducts. Moreover, delicate VAChT-immunoreactive (-Ir) nerve fibers were observed on the periphery of the pancreatic islets. $\mathrm{D} \beta \mathrm{H}$-positive and $\mathrm{TH}$-positive nerve fibers between pancreatic parenchyma acini and in the wall of blood vessels were visible (Fig. 1E-H). The walls of the blood vessels were dominated by $\mathrm{D} \beta \mathrm{H}$-positive fibers that formed a distinct network (Fig. 1F). Immunoreactive ChAT and $\mathrm{D} \beta \mathrm{H}$ fibers were found in the area of the Langerhans islets (Fig. 1G). Double labeling with ChAT/ $/ \mathrm{D} \beta \mathrm{H}$ revealed colocalization of these markers in the parenchymal fibers and in the fibers passing through the cholinergic ganglia (Fig. 1E-F). Individual nerve cells and very small ganglia consisting of 2 to 3 cells were also immunoreactive for both ChAT and $\mathrm{D} \beta \mathrm{H}$. Colocalization of VAChT and TH was found in the nerve fibers of the exocrine part, in the blood vessel walls, and in individual nerve cells (Fig. 1H). The activity of ChAT and VAChT was observed mainly in pancreas head and tail, and $\mathrm{D} \beta \mathrm{H}+$ and $\mathrm{TH}$-positive structures were evenly distributed in each part of the investigated organ.

No sex-related differences were observed in the innervation of chinchilla's pancreas.

\section{Discussion}

According to various classical investigations, intrapancreatic nerve fibers generally form some plexuses: peri-ductal, peri-vascular, peri-acinar, and peri-insular. Similar, but not identical, distribution of the pancreatic innervation was observed in different groups of vertebrates.

In cold-blood animals, cholinergic innervation of the pancreas has been observed in a few amphibian and reptile species. In amphibians, AChE-positive nerve fibers have been found in the pancreatic parenchyma running along the blood vessels and in the interlobular connective tissue, but only a few reach the pancreatic acini and islets of Langerhans. The cholinergic fibers formed a delicate network as the intra- and peri-insular plexuses in frogs [9]. Cholinergic innervation of the exocrine part of the pancreas has been observed in lizards in which nerve fibers were found in the wall of blood vessels and pancreatic duct and wrapped pancreatic acini. In snakes, the density of cholinergic innervation of the pancreas is low and has mainly been observed in the interlobular connective tissue, along the blood vessels, and rarely between the acinar cells. In reptiles, sporadic cholinergic fibers have been found in the islets of Langerhans [9]. Compared to the studied species, which had numerous fibers and ganglionic cells within the exocrine part of the pancreas, only AChE-positive nerve fibers were found in amphibians and reptiles. In general, cholinergic innervation in lower vertebrates is definitely less developed compared to higher vertebrates. Results of the investigations on the adrenergic innervation of pancreas in amphibian and reptiles are not accessible in scientific databases [30].

A review of the literature indicates that cholinergic structures of birds and mammals consist of numerous nerve fibers and ganglia while the adrenergic innervation consists mainly of nerve fibers as found in most investigated species. Investigations carried out in hen revealed that cholinergic and adrenergic nerve fibers and bundles of differing thickness supply the pancreas along with blood vessels that form the perivascular plexus [11]. Some of these accompany the pancreatic ducts. Moreover, numerous cholinergic fibers have been observed under the connective tissue capsule of the pancreas. Both types of nerve fiber accompany the interlobar and interlobular arteries as the perivascular plexuses run in the connective tissue, and their numerous branches reached the pancreatic acini and islets. Individual ChAT- and TH-positive neurocytes were found in the pancreatic parenchyma [11].

Similar results regarding pancreatic cholinergic innervation were reported in the investigation of other species of birds, such as the domestic pigeon and African silverbill $[9,10]$. The nerve fibers observed in the parenchyma form a perivascular plexus that supplies the exocrine parts of the pancreas. Moreover, small agglomerations of neurocytes were found mainly on the gland surface and in its immediate vicinity with less inside the gland. Only individual neurons were observed near the blood vessels, nerve fibers, and pancreatic ducts. Islet innervation is relatively weak, which suggests the presence of delicate fibers that wrap themselves around the periphery [10].

Cholinergic innervation of the chinchilla pancreas is better developed compared to investigated bird species. Agglomerations of neurocytes in this species form several-cell ganglia and are distributed throughout the entire organ. In contrast, adrenergic structures, as in birds, are single neurocytes and numerous nerve fibers.

Investigations involving rat, cat and rabbit have shown cholinergic fibers running in the interlobular connective tissue forming perivascular plexuses, the branches of which reach the pancreatic parenchyma. Numerous delicate nerve fibers were dispersed among the pancreatic acini forming peri-acinar and -insular plexuses that supply the islets of Langerhans [12]. Additionally, investigations conducted in rabbit revealed the presence of AChE-positive ganglionic cells except 
cholinergic nerve fibers. Individual neurocytes and small ganglia were visible in the interlobular connective tissue. Characteristic $\mathrm{D} \beta \mathrm{H}$-positive networks were seen in the wall of the blood vessels, and individual fibers were observed around neurons [13].

The examination of adrenergic innervation in rat has revealed small $\mathrm{TH}$-immunoreactive nerve cells in close contact with large TH-immuno-negative ganglionic neurocytes in the exocrine part of the pancreas and occasionally in the islets. Some of these $\mathrm{TH}$ - immunoreactive cells were also $\mathrm{D} \beta \mathrm{H}$-immuno-positive. All intrapancreatic ganglion cells were immunoreactive for $\mathrm{D} \beta \mathrm{H}$ but not for $\mathrm{TH}$. $\mathrm{TH}$-immunoreactive nerve fibers were found in both the exocrine and endocrine parts of the pancreas [17].

Investigations performed on the mouse pancreas revealed thick AChE-positive nerve fibers in the interlobular connective tissue in which they formed perivascular plexuses. At the end of the vascular segment, fibers left vessels and formed a loosely organized network between the acini, which also sent off delicate branches toward the islets of Langerhans [6]. Moreover, immunohistochemical staining showed $\mathrm{TH}$-positive nerve fibers in the exocrine part forming delicate network around blood vessels and adrenergic nerve fibers in the area of Langerhans islets [5]. Similar distribution of the cholinergic fibers was found in the pancreas of the Egyptian spiny mouse in which fibers along the pancreatic ducts and branches of pancreaticoduodenal arteries reached the Langerhans islets. AChE-positive ganglia were observed throughout the exocrine part and fine adrenergic fibers accompanied the blood vessels [19].

Studies of sheep pancreas revealed a moderate number of VAChT-positive nerve endings among the pancreatic acini, while only a few cholinergic fibers were found in the interlobular connective tissue. Similarly, a small number of VAChT-immunoreactive fibers supplied the endocrine part of the pancreas. VAChT and TH were shown to be colocalized in distinct populations of nerve fibers among the acini. TH was present in the vast majority of VAChT-positive intrapancreatic perikarya [14]. Studies of adrenergic pancreatic innervation in sheep revealed that most $\mathrm{D} \beta \mathrm{H}$-positive nerve fibers occur in the parenchyma between the acini, around blood vessels, and in the connective tissue, but only a small amount of adrenergic intrapancreatic neurons were found. Moreover, delicate $\mathrm{D} \beta \mathrm{H}$-immunoreactive nerve fibers were seen around the islets of Langerhans, of which only a few penetrated between $\mathrm{D} \beta \mathrm{H}$-negative endocrine cells [15].

Investigations of the pancreas of sow showed cholinergic structures as perivascular plexuses, inter-lobular nerve fibers, peri-lobular and -insular plexuses throughout the organ and as individual cholinergic neurons in the parenchyma [16].

Studies of the pancreas of newborn guinea pig revealed ChAT-immunoreactive ganglia in the interlobular connective tissue, between the acini, and in close proximity to the islets of Langerhans, blood vessels, and pancreatic ducts. Moreover, double staining showed the presence of ChAT and TH in the same ganglionic cells. ChAT-immunopositive nerve fibers were distributed as thick bundles running adjacent to blood vessels in the interlobular connective tissue, fine fibers in the adventitia of blood vessels, individual fiber bundles running between the acini throughout the whole organ or occasionally as small bundles near the periphery of the islets of Langerhans [31].

Similar results were obtained for pancreatic innervation in the Dromedary camel. Cholinergic fibers were observed in the parenchyma, around the pancreatic acini forming peri-acinar, -ductal, and -insular plexuses. Nerve cells were sporadically noted in close proximity or within islands. Adrenergic nerve fibers with characteristic varicosities were also observed to accompany blood vessels and interlobular ducts. Delicate adrenergic fiber ends were found in close proximity to the islets [32].

The results of our investigations on the autonomic innervation of chinchilla's pancreas indicate similar findings as seen in laboratory rodents, including the presence of distinctive nerve plexuses surrounding blood vessels and pancreatic ducts and acini. Forming the ganglia, agglomerations of the neurocytes mainly had cholinergic properties, whereas the adrenergic structures consisted of only fine nerve fibers and individual neurocytes. Moreover, our investigation indicates that the nerve plexuses in the chinchilla's pancreas are distinctly dense around the blood vessels but are loosely organized around pancreatic ducts and acini. This type of distribution of the pancreatic innervation in the studied species indicates a clear domination of cholinergic over adrenergic structures.

\section{References}

1. Löve JA, Yi E, Smith TG. Autonomic pathways regulating pancreatic exocrine secretion. Auton Neurosci. 2007; 133(1): 19-34, doi: 10.1016/j.autneu.2006.10.001, indexed in Pubmed: 17113358

2. Ahrén B. Autonomic regulation of islet hormone secretion — implications for health and disease. Diabetologia. 2000; 43(4): 393-410, doi: 10.1007/s001250051322, indexed in $\mathrm{Pu}-$ bmed: 10819232 .

3. Gilon P, Henquin JC. Mechanisms and physiological significance of the cholinergic control of pancreatic beta-cell function. Endocr Rev. 2001; 22(5): 565-604, doi: 10.1210/ edrv.22.5.0440, indexed in Pubmed: 11588141.

4. Rodriguez-Diaz R, Abdulreda MH, Formoso AL, et al. Innervation patterns of autonomic axons in the human endo- 
crine pancreas. Cell Metab. 2011; 14(1): 45-54, doi: 10.1016/j. cmet.2011.05.008, indexed in Pubmed: 21723503.

5. Lindsay TH, Halvorson KG, Peters CM, et al. A quantitative analysis of the sensory and sympathetic innervation of the mouse pancreas. Neuroscience. 2006; 137(4): 1417-1426, doi: 10.1016/j.neuroscience.2005.10.055, indexed in Pubmed: 16388907.

6. Ushiki T, Watanabe S. Distribution and ultrastructure of the autonomic nerves in the mouse pancreas. Microscopy Research and Technique. 1997; 37(5-6): 399-406, doi: 10.1002/ (sici)1097-0029(19970601)37:5/6<399::aid-jemt4>3.0.co;2-9.

7. Babic T, Travagli RA. 2016. Neuronal control of the pancreas. Pancreapedia. Exocrine pancreas knowledge base. Version 1; 0: September, doi: 10.3998/panc.2016.27.

8. Li W, Yu G, Liu Y, et al. Intrapancreatic ganglia and neural regulation of pancreatic endocrine secretion. Front Neurosci. 2019; 13: 21, doi: 10.3389/fnins.2019.00021, indexed in Pubmed: 30842720

9. Trandaburu T. Comparative observations on AChE distribution in pancreas of some amphibians, repitiles and birds, with special reference to the islets of langerhans. Histochemie. 1972; 32(3): 271-279, doi: 10.1007/bf00306034, indexed in Pubmed: 4562919.

10. Trandaburu T. Ultrastructural and acetylcholinesterase investigations on the pancreas intrinsic innervation of two bird species (Columba livia domestica Gm. and Euodice cantans Gm.). Gegenbaurs Morphol Jahrb. 1974; 120(6): 888-904, indexed in Pubmed: 4618821.

11. Ulas M, Penkowski A, Lakomy M. Adrenergic and cholinergic innervation of the chicken pancreas. Folia Morphol (Warsz). 2003; 62(3): 243-246, indexed in Pubmed: 14507057.

12. COUPLAND RE. The innervation of pan creas of the rat, cat and rabbit as revealed by the cholinesterase technique. J Anat. 1958; 92(1): 143-149, indexed in Pubmed: 13513506.

13. Löve JA, Szebeni K. Morphology and histochemistry of the rabbit pancreatic innervation. Pancreas. 1999; 18(1): 53-64, doi: 10.1097/00006676-199901000-00008, indexed in Pubmed: 9888661.

14. Arciszewski MB, Zacharko-Siembida A. Cholinergic innervation of the pancreas in the sheep. Acta Biol Hung. 2007; 58(2): 151-161, doi: 10.1556/ABiol.58.2007.2.2, indexed in Pubmed: 17585505 .

15. Arciszewski MB, Zacharko-Siembida A. A co-localization study on the ovine pancreas innervation. Ann Anat. 2007; 189(2): 157-167, doi: 10.1016/j.aanat.2006.09.002, indexed in Pubmed: 17419548.

16. Lakomy M, Chodkowska D. Cholinergic innervation of pig pancreas. Acta Histochem. 1984; 75(1): 63-68, doi: 10.1016/ S0065-1281(84)80072-0, indexed in Pubmed: 6209910.

17. Oomori $\mathrm{Y}$, Iuchi $\mathrm{H}$, Ishikawa $\mathrm{K}$, et al. Immunocytochemical study of tyrosine hydroxylase and dopamine beta-hydroxylase immunoreactivities in the rat pancreas. Histochemistry. 1994; 101(5): 313-323, doi: 10.1007/bf00268992, indexed in Pubmed: 7523336.

18. Gienc J, Kosierkiewicz D, Kuder T. Ganglionic cells and their localization within the secretory system of pancreas in vertebrates. Zool Pol. 1993; 38: 27-38.
19. Szczurkowski A, Kuchinka J, Nowak E, et al. Autonomic innervation of pancreas in egyptian spiny mouse (acomys cahirinus, desmarest). Acta Veterinaria Brno. 2009; 78(4): 557-561, doi: 10.2754/avb200978040557.

20. Fabris SE, Thorburn A, Litchfield A, et al. Effect of parasympathetic denervation of liver and pancreas on glucose kinetics in man. Metabolism. 1996; 45(8): 987-991, doi: 10.1016/s00260495(96)90268-1, indexed in Pubmed: 8769357.

21. Suckow M A, Stevens K A, Wilson R P. The laboratory rabbit, guinea pig, hamster, and other rodents. 1st ed. Saunders: Elsevier 2012.

22. Kuchinka J. Morphometry and variability of the brain arterial circle in chinchilla (Chinchilla laniger, Molina). Anat Rec (Hoboken). 2017; 300(8): 1472-1480, doi: 10.1002/ar.23566, indexed in Pubmed: 28181413.

23. Nowak E. Organization of the innervation of the oesophagus and stomach in chinchilla (Chinchilla laniger, Molina). Folia Histochem Cytobiol. 2013; 51(2): 115-120, doi: 10.5603/ fhc.2013.0018.

24. Nowak E. Organisation of autonomic nervous structures in the large intestine of chinchilla (Chinchilla laniger Molina). Folia Biol (Krakow). 2013; 61(3-4): 135-141, doi: 10.3409/ fb61_3-4.135, indexed in Pubmed: 24279160.

25. Nowak E. Organisation of autonomic nervous structures in the small intestine of chinchilla (Chinchilla laniger, Molina). Anat Histol Embryol. 2014; 43(4): 301-309, doi: 10.1111/ ahe.12077, indexed in Pubmed: 23848953.

26. Nowak E, Kuchinka J, Szczurkowski A, et al. Extrahepatic biliary tract in chinchilla (Chinchilla laniger, Molina). Anat Histol Embryol. 2015; 44(3): 236-240, doi: 10.1111/ahe.12137, indexed in Pubmed: 25091180.

27. Karnovsky MJ, Roots L. A "direct-coloring" thiocholine method for cholinesterases. J Histochem Cytochem. 1964; 12: 219-221, doi: 10.1177/12.3.219, indexed in Pubmed: 14187330.

28. Tsuji S, Larabi Y. A modification of thiocholine-ferricyanide method of Karnovsky and Roots for localization of acetylcholinesterase activity without interference by Koelle's copper thiocholine iodide precipitate. Histochemistry. 1983; 78(3): 317-323, doi: 10.1007/bf00496619, indexed in Pubmed: 6193086.

29. De la Torre JC. An improved approach to histofluorescence using the SPG method for tissue monoamines. J Neurosci Methods. 1980; 3(1): 1-5, doi: 10.1016/0165-0270(80)90029-1, indexed in Pubmed: 6164878.

30. Trandaburu T. Comparative observations on adrenergic innervation and monoamine content in endocrine pancreas of some amphibians, reptiles and birds. Endokrinologie. 1972; 59(2): 260-264, indexed in Pubmed: 4556423.

31. Liu HP, Tay SS, Leong S, et al. Colocalization of ChAT, DbetaH and NADPH-d in the pancreatic neurons of the newborn guinea pig. Cell Tissue Res. 1998; 294(2): 227-231, doi: 10.1007/s004410051172, indexed in Pubmed: 9799438.

32. Qayyum MA, Fatani JA, Shaad FU, et al. A histochemical study on the innervation of the pancreas of the one-humped camel (Camelus dromedarius). J Anat. 1987; 151: 117-123, indexed in Pubmed: 3654346.

Submitted: 6 November, 2019

Accepted after reviews: 16 March, 2020 Available as AoP: 23 March, 2020 